\title{
Tratamiento de inducción y mantenimiento con adalimumab en la enfermedad de Crohn: un estudio abierto
}

\author{
N. López Palacios, J. L. Mendoza, C. Taxonera, R. Lana ${ }^{1}$, M. Fuentes Ferrer ${ }^{2}$ y M. Díaz-Rubio

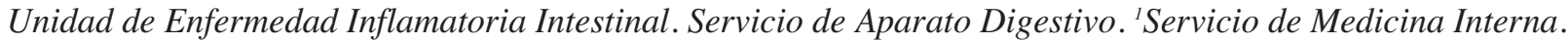 \\ ${ }^{2}$ Servicio de Estadística. Hospital Clínico San Carlos. Madrid
}

\section{RESUMEN}

Introducción: el adalimumab ha demostrado, en ensayos clínicos controlados con placebo y en estudios no controlados, ser efectivo en la EC luminal y fistulosa perianal.

Objetivo: evaluar la eficacia y seguridad del adalimumab como tratamiento de inducción y mantenimiento en la EC.

Metodología: se incluyeron 22 pacientes con EC tratados con adalimumab (16 por enfermedad luminal y 6 por enfermedad fistulosa perianal activa). Veintiún pacientes habían recibido previamente IFX. Se realizó tratamiento de inducción con $160 \mathrm{mg}$ s.c. en la semana 0 y $80 \mathrm{mg}$ s.c. a las 2 semanas. Los respondedores recibieron $40 \mathrm{mg}$ s.c. cada 14 días como tratamiento de mantenimiento. Se valoró la respuesta a las 4 semanas de la dosis inicial, y se clasificó la respuesta como remisión, respuesta parcial o ausencia de respuesta.

Resultados: tras la inducción, el 25\% de los pacientes con enfermedad luminal tuvieron remisión completa y el 56,3\% respuesta parcial. La respuesta clínica se mantuvo al año en el $71,6 \%$ de los pacientes, a los 18 meses en el 53,7\% y a los 48 meses en el $35,8 \%$. No se objetivaron diferencias en la respuesta entre pacientes que presentaron reacciones de hipersensibilidad o pérdida de respuesta a IFX.

Todos los pacientes con enfermedad fistulosa perianal $(n=6)$ habían recibido previamente tratamiento con IFX. Tras la inducción un $16,7 \%$ entran en remisión y un $66,7 \%$ presentan respuesta parcial. Todos los pacientes mantienen remisión o respuesta en el tiempo con una mediana de seguimiento de 15 meses.

Conclusiones: el adalimumab es un tratamiento eficaz y seguro en la inducción y mantenimiento de la respuesta en la EC luminal y fistulosa perianal. Estos resultados confirman que los hallazgos obtenidos en los ensayos clínicos controlados son reproducibles en la práctica clínica diaria.

Palabras clave: Adalimumab. Tratamiento de mantenimiento. Enfermedad de Crohn. Luminal. Enfermedad fistulosa perianal.

\begin{abstract}
Background: adalimumab has been shown in placebo-controlled clinical trials and uncontrolled studies to be effective in luminal and perianal fistulizing CD.

Objective: to evaluate the efficacy and safety of adalimumab for induction and maintenance therapy in $C D$.

Methods: twenty-two patients with $\mathrm{CD}$ treated with adalimumab (16 for luminal disease and 6 for active perianal fistulizing disease) were included. Twenty-one patients had previously received IFX. All patients received induction therapy with $160 \mathrm{mg}$ s.c. at week 0 , and $80 \mathrm{mg}$ s.c. at week 2. Responders received maintenance therapy with $40 \mathrm{mg}$ s.c. every 14 days. Response was assessed at 4 weeks after the initial dose, and classified as remission, partial response, or non-response.

Results: after induction, 25\% of patients with luminal disease had a complete remission, and $56.3 \%$ had a partial response. Clinical response was maintained in $71.6 \%$ of patients at 1 year, in $53.7 \%$ at 18 months, and in $35.8 \%$ at 48 months. No differences in response were observed between patients with hypersensitivity reactions or loss of response to IFX.

All patients with perianal fistulizing disease $(n=6)$ had been previously treated with IFX. After induction $16.7 \%$ entered remission, and $66.7 \%$ had a partial response. All patients maintained remission or response over time, with a median follow-up of 15 months.

Conclusions: adalimumab is an effective and safe treatment for the induction and maintenance of response in luminal and perianal fistulizing $\mathrm{CD}$. These results confirm that the findings obtained in controlled clinical trials are reproducible in clinical practice.
\end{abstract}

Key words: Adalimumab. Maintenance therapy. Crohn's disease. Luminal. Perianal fistulizing disease.

López Palacios N, Mendoza JL, Taxonera C, Lana R, Fuentes Ferrer M, Díaz-Rubio M. Tratamiento de inducción y mantenimiento con adalimumab en la enfermedad de Crohn: un estudio abierto. Rev Esp Enferm Dig 2008; 100: 676-681. 


\section{INTRODUCCIÓN}

El adalimumab es un anticuerpo monoclonal IgG1 recombinante completamente humano que se une con gran afinidad y especificidad al factor de necrosis tumoral alfa $(\mathrm{TNF} \alpha)$ tanto soluble como unido a la membrana celular $(1,2)$. Recientemente, el adalimumab ha demostrado ser efectivo como tratamiento de inducción en la enfermedad de Crohn $(\mathrm{EC})$ con brote moderado-grave $(3,4)$ y como tratamiento de mantenimiento de la remisión $(3,5)$. Además, ha demostrado ser eficaz tanto en pacientes naüve para antiTNF $\alpha$ (3) como en aquellos con pérdida de respuesta o intolerancia al infliximab (IFX) (6).

El TNF $\alpha$ es una citoquina proinflamatoria implicada en la fisiopatología de la enfermedad inflamatoria intestinal (EII). El primer agente antiTNF $\alpha$ que demostró su eficacia en la EC con brote inflamatorio o fistuloso fue el $\operatorname{IFX}(7,8)$, un anticuerpo quimérico monoclonal antiTNFa. El IFX posee una fracción murina del $25 \%$, que se ha relacionado con la aparición de inmunogenicidad, hecho que acaece en el $30 \%$ de los pacientes (9). La presencia de anticuerpos antiinfliximab (ATI) se ha asociado con la pérdida de respuesta a este fármaco y con la aparición de reacciones de hipersensibilidad aguda y retardada que obligan en ocasiones a suspender el tratamiento (7). Los ATI aparecen con mayor frecuencia cuando se utiliza el IFX de forma episódica y no asociado a inmunosupresores (10). La pérdida de respuesta al IFX es un problema complejo, lo que deja hueco para el desarrollo de nuevos fármacos antiTNFo que no tengan inmunogenicidad cruzada con IFX, entre los que se encuentra el adalimumab. Este antiTNF $\alpha$ totalmente humano desencadena inmunogenicidad $(4,5)$, pero el papel que los anticuerpos anti-adalimumab tienen en la pérdida de respuesta y las posibles reacciones de hipersensibilidad se desconocen.

\section{OBJETIVO}

El objetivo de este trabajo es valorar, en una serie de pacientes consecutivos de un único centro, la eficacia y seguridad del tratamiento con adalimumab en la EC.

\section{MATERIAL Y MÉTODOS}

Estudio descriptivo, longitudinal, abierto y retrospectivo de seguimiento (eficacia y seguridad) en pacientes con EC tratados con adalimumab.

Se incluyeron de forma consecutiva 22 pacientes con EC diagnosticados por criterios clínicos, endoscópicos e histológicos y que recibieron tratamiento con adalimumab en el Hospital Clínico San Carlos de Madrid entre marzo de 2004 y diciembre de 2007.

De los 22 pacientes, 8 son hombres y 14 mujeres, con una mediana de edad al diagnóstico de 23,7 años (RIQ: $15,2-30,5)$ y con una mediana de seguimiento desde el diagnóstico de 13,1 años (RIQ 6,5-18,7). Veintiún pacientes habían recibido previamente IFX (Tabla I). Dieciséis pacientes recibieron adalimumab por enfermedad luminal y 6 por enfermedad fistulosa perianal activa (Tabla II).

En todos los pacientes se realizó tratamiento de inducción a dosis de $160 \mathrm{mg}$ subcutáneo (s.c.) en la semana 0 , seguido de $80 \mathrm{mg}$ s.c. a las 2 semanas. Los respondedores recibieron un tratamiento de mantenimiento con $40 \mathrm{mg}$ s.c. cada 14 días. En caso de pérdida de respuesta se acortó el intervalo entre dosis recibiendo $40 \mathrm{mg} \mathrm{s.c.} \mathrm{cada} \mathrm{se-}$ mana. Las reacciones adversas al tratamiento durante el periodo de seguimiento fueron recogidas y anotadas en la historia clínica.

Tabla I. Características basales de los pacientes con EC que recibieron adalimumab

\begin{tabular}{lc}
\hline Variables & Resultados \\
\hline Sexo & \\
Hombre/mujer & $8: 14$ \\
\hline Edad al diagnóstico (años) & $23,71$ (RIQ: $15,24-30-53)$ \\
Tiempo pasado desde diagnóstico & \\
a inicio terapia adalimumab (años) & $13,08$ (RIQ $6,53-18,69)$ \\
\hline Clasificación de Montreal & \\
Edad al diagnóstico & \\
$0-15$ años & $6(27,3 \%)$ \\
16-40 años & $15(68,2 \%)$ \\
$\geq 40$ años & $1(4,5 \%)$ \\
Localización & \\
Ileon & $6(27,3 \%)$ \\
Colon & $4(18,2 \%)$ \\
Illeo-colónica & $10(45,5 \%)$ \\
Tracto superior & $2(9,1 \%)$ \\
Comportamiento & \\
Inflamatorio & $6(27,3 \%)$ \\
Estenosante & $2(9,1 \%)$ \\
Penetrante & $5(22,7 \%)$ \\
Inflamatorio + perianal & $4(18,25)$ \\
Estenosante + perianal & $1(4,5 \%)$ \\
Penetrante + perianal & $4(18,2 \%)$ \\
\hline
\end{tabular}

Indice de Harvey-Branshaw

mediana [rango] grupo luminal $(n=17)$

10 (RIQ 7-12,5)

Afectación perianal asociada

$\mathrm{n}(\%) \quad 9(41 \%)$

Cirugía previa

$\mathrm{n}(\%) \quad 15(68,2)$

Resección $12(54,5 \%)$

$\begin{array}{ll}\text { Cirugía perianal } & 8(36,4 \%)\end{array}$

Medicación concomitante: inmunosupresores $\mathrm{n}(\%)$ $18(81,8 \%)$

Tratamiento antiTNF $n$ (\%)

Naive antiTNF

$1(4,5 \%)$

Tratamiento con infliximab

Administración episódica

Motivo suspensión de la medicación

Pérdida de respuesta

$21(95,5 \%)$

$4(18,19 \%)$

Reacción de hipersensibilidad

$8(36,38 \%)$

Pérdida de respuesta + hipersensibilidad
$8(36,38 \%)$

$1(4,55 \%)$ 
Tabla II. Características basales de los pacientes con EC que recibieron adalimumab según su indicación

\begin{tabular}{lcc}
\hline Variables & EC luminal & EC fistulosa perianal \\
\hline Número de pacientes & 16 & 6 \\
Edad al diagnóstico mediana (RIQ) & $21,27(15,23-27,34)$ & $35,32(21,46-40,23)$ \\
Tiempo desde diagnóstico al tratamiento & $11,21(6,18-15,52)$ & $19,35(9,11-32,23)$ \\
Sexo: hombres/mujeres & $5: 11$ & $3: 3$ \\
Clasificación de Montreal n (\%) & & \\
Edad & & \\
0-15 años & & \\
16-40 años & $5(31,3 \%)$ & $1(16,7 \%)$ \\
$>40$ años & $11(68,8 \%)$ & $4(66,7 \%)$ \\
Localización & - & $1(16,7 \%)$ \\
Illeon & $3(18,8 \%)$ & $3(50 \%)$ \\
Colon & $3(18,8 \%)$ & $1(16,7 \%)$ \\
Illeon-colon & $8(50 \%)$ & $2(33,3 \%)$ \\
Tracto superior & $2(12,5 \%)$ & - \\
Comportamiento & & \\
Inflamatorio & $6(37,5 \%)$ & - \\
Estenosante & $2(12,5 \%)$ & - \\
Penetrante & $5(31,3 \%)$ & - \\
Inflamatorio + perianal & $1(6,3 \%)$ & $3(50 \%)$ \\
Estenosante + perianal & $1(6,3 \%)$ & - \\
Penetrante + perianal & $1(6,3 \%)$ & $3(50 \%)$ \\
IHB mediana (RIQ) & $9,50(7-12,75)$ & - \\
Cirugía n (\%) & $9(56,3 \%)$ & $6(100 \%)$ \\
Resección quirúrgica n (\%) & $7(43,8 \%)$ & $5(83,3 \%)$ \\
Mediación concomitante: inmunosupresor & $13(81,3 \%)$ & $5(83,3 \%)$ \\
Infliximab & $15(93,8 \%)$ & $6(100 \%)$ \\
Pérdida de respuesta & $4(25,01 \%)$ & $4(66,7 \%)$ \\
Reacción de hipersensibilidad & $6(37,52 \%)$ & $2(33,3 \%)$ \\
Pérdida de respuesta + & $1(6,25 \%)$ & - \\
reacción de hipersensibilidad & $4(25,01 \%)$ & - \\
Administración episódica & \\
\hline
\end{tabular}

La pérdida de eficacia al IFX se definió como la falta de respuesta tras acortar el intervalo entre las infusiones a menos de 6 semanas o tras aumentar la dosis a $10 \mathrm{mg} / \mathrm{kg}$ peso. Se consideró reacción de hipersensibilidad aguda a IFX a la ocurrida durante la infusión o en las 2 horas posteriores, definida como la existencia de: hipotensión, urticaria, rash cutáneo, edema (facial, de manos, labial o bucal), cefalea o disnea. La reacción de hipersensibilidad retardada se definió como la existencia de al menos 2 de los siguientes signos o síntomas ocurridos dentro de los primeros días después de la infusión: rash, fiebre, mialgias o poliartralgias.

La administración episódica de IFX se define como la administración de este fármaco a demanda según el paciente presentaba recidiva de los síntomas.

A todos los pacientes se les realizó una historia clínica de contacto de tuberculosis, Mantoux y booster, radiografía de tórax, anticuerpos antinucleares y anti-DNA de doble cadena y serología de VHB y VHC, con resultados negativos en todos ellos.
El seguimiento clínico se realizó con visitas periódicas en la unidad de EII, cada 15 días los 2 primeros meses, 1 vez al mes los siguientes 3 meses y posteriormente cada 2 meses hasta el final del seguimiento. La respuesta a la inducción se valoró a las 4 semanas de la dosis inicial de adalimumab.

La respuesta se clasificó como remisión, respuesta parcial o ausencia de respuesta. La respuesta en la EC luminal se evaluó según el índice de Harvey-Brandshaw (IHB) (11). Se definió remisión como el cese del dolor abdominal, la diarrea y la mejoría del estado general, con un IHB menor o igual a 4 , y respuesta parcial como la mejoría incompleta con un descenso de 4 o más puntos en el IHB con respecto a la basal. El resto de las situaciones se consideraron no respuesta al tratamiento.

En la enfermedad fistulosa perianal se evaluó la respuesta de acuerdo con el número de fístulas, el drenaje a través de estas y la existencia o no de dolor en la zona perianal. Se definió la remisión como el cierre total de todas las fístulas con la ausencia de drenaje, y la respuesta parcial como la reducción en el número, forma, drenaje y molestias asociadas con las fístulas.

Todos los pacientes firmaron el consentimiento informado para la administración en uso compasivo de adalimumab.

\section{Análisis estadístico}

Las variables cualitativas se presentan con su distribución de frecuencias. Las variables cuantitativas se resumen en su media y desviación estándar (DE) o su mediana y rango intercuartílico (RIQ) para variables que no siguen una distribución normal.

Se evalúa la asociación entre variables cualitativas con el test de $\chi^{2}$ o prueba exacta de Fisher, en el caso de que más de un $25 \%$ de los esperados sean menores de 5 . Se estimaron las funciones de supervivencia por el método de Kaplan-Meier para evaluar el motivo (perianal e inflamatorio) para el estudio del evento respuesta al tratamiento. Se presentan las gráficas de las curvas estimadas y la mediana de la distribución junto a su intervalo de confianza. La comparación de las funciones de supervivencia de los distintos subgrupos se realizó mediante el test exacto de Breslow.

En todos los contrastes de hipótesis se rechazó la hipótesis nula con un error de tipo I o error $\alpha$ menor a 0,05 . El paquete informático utilizado para el análisis fue SPSS para Windows versión 15.0.

\section{RESULTADOS}

\section{Eficacia del tratamiento con adalimumab}

\section{Tratamiento de inducción en la enfermedad luminal}

En la semana 4 de los 16 pacientes con enfermedad luminal que recibieron tratamiento de inducción con adalimumab en cuatro pacientes $(25 \%)$ se obtiene la re- 
misión y en $9(56,3 \%)$ respuesta parcial. Durante el tratamiento de inducción fue necesaria la retirada precoz de un paciente que precisó cirugía por obstrucción intestinal.

De los quince pacientes tratados previamente con IFX, 3 pacientes (20\%) entran en remisión y en $9(66,7 \%)$ se produce respuesta parcial. Seis de estos pacientes habían presentado reacciones de hipersensibilidad a IFX, 4 pérdida de respuesta, 1 pérdida de respuesta e hipersensibilidad y 4 habían recibido IFX en administración episódica.

\section{Tratamiento de inducción en la enfermedad fistulosa perianal}

En la semana 4, de los 6 pacientes con enfermedad fistulosa perianal activa, un paciente $(16,7 \%)$ entra en remisión y $4(66,7 \%)$ presentan respuesta parcial. Todos los pacientes habían recibido previamente IFX (4 pacientes pertenecían al grupo de pérdida de respuesta y 2 al grupo de reacción de hipersensibilidad).

\section{Tratamiento de mantenimiento en la enfermedad luminal}

Trece pacientes (12 de ellos tratados previamente con IFX) continuaron con el tratamiento de mantenimiento con una mediana de tiempo de seguimiento de 12,6 meses (RIQ 4,6-16,8). El porcentaje de pacientes que mantuvieron la respuesta a los 6 meses fue del 81,8\% (IC 95\% 45-95\%), a los doce meses del 71,6\% (IC 95\% 35-90\%), a los 18 meses del $53,7 \%$ (IC $95 \% 15-81 \%$ ) y a los 24 meses del 35,8\% (IC 95\% 5-69\%) (Tabla III y Fig. 1).

Seis pacientes $(27,3 \%)$ requirieron acortar el intervalo entre dosis tras un seguimiento de 9,2 meses de mediana (RIQ 3,1-13,4). Cuatro mantienen respuesta con $40 \mathrm{mg}$ de adalimumab semanal, mientras dos de ellos pierden definitivamente la respuesta a los 2 meses de acortar el intervalo.

Tabla III. Porcentaje de pacientes con EC luminal que mantienen respuesta o remisión en el tiempo

\begin{tabular}{lcc}
\hline \multirow{2}{*}{ Tiempo } & \multicolumn{2}{c}{ Porcentaje de respuesta/remisión } \\
\cline { 2 - 3 } & $\%$ & IC 95\% \\
\hline 6 meses & 81,8 & $45-95$ \\
12 meses & 71,6 & $35-90$ \\
18 meses & 53,7 & $15-81$ \\
24 meses & 35,8 & $5-69$ \\
\hline
\end{tabular}

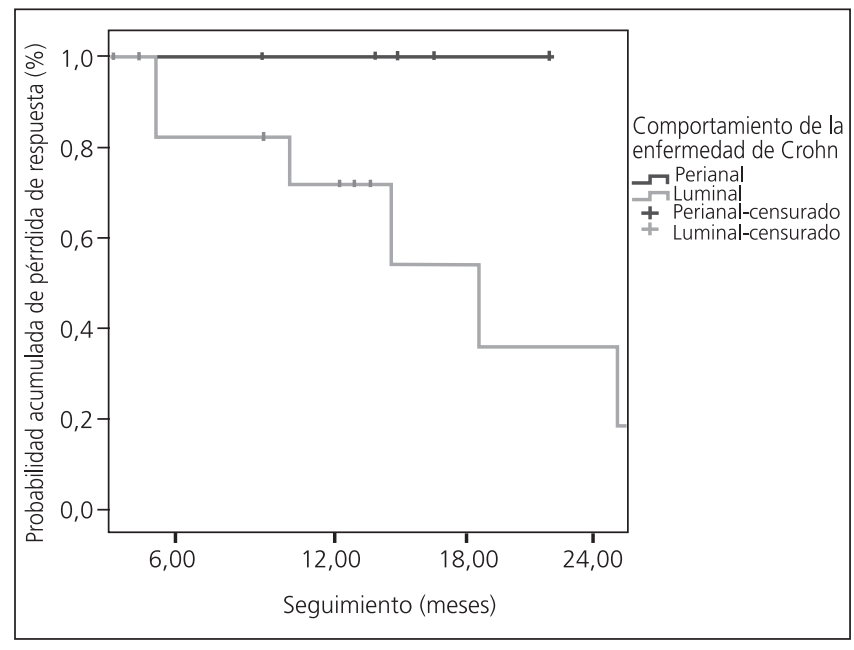

Fig. 1. Probabilidad acumulada de pérdida de respuesta en el tiempo.

\section{Tratamiento de mantenimiento en la enfermedad fisulosa perianal}

Cinco pacientes ( 3 con pérdida de eficacia a IFX y 2 reacciones de hipersensibilidad) continuaron con el tratamiento de mantenimiento con una mediana de tiempo de seguimiento de 15,2 meses (RIQ 11,7-19,4). Todos ellos mantienen remisión o respuesta en el tiempo.

\section{Tolerancia y efectos adversos al tratamiento con adalimumab}

Siete pacientes $(31,8 \%)$ desarrollaron efectos secundarios. Cuatro de ellos presentaron reacción local en la zona de la inyección sin necesidad de suspender el tratamiento. En un paciente fue necesaria la suspensión del tratamiento por complicaciones de la EC: una obstrucción intestinal que requirió cirugía. En otro caso se produjo una nasofaringitis aguda que se resolvió con tratamiento antibiótico convencional.

\section{DISCUSIÓN}

El adalimumab ha demostrado en ensayos clínicos controlados con placebo (3-6) y en estudios no controlados (12-16) que es eficaz como tratamiento de inducción y mantenimiento en la EC luminal y fistulosa perianal. Nuestro trabajo muestra que en la práctica clínica habitual el adalimumab puede ser eficaz en pacientes con EC que han presentado pérdida de respuesta o intolerancia a IFX.

Los primeros datos publicados sobre la eficacia del adalimumab en la EC luminal en pacientes que han re- 
cibido previamente IFX son estudios abiertos no controlados (12-16). En el estudio de Sandborn y cols. (16) con dosis de inducción de $80 / 40 \mathrm{mg}$, se obtiene remisión en el $12 \%$ de los pacientes y respuesta parcial en el $41 \%$. En el estudio de Hinojosa y cols. (13) con inducción a dosis de 160/80 mg, las tasas de remisión y respuesta obtenidas son del 42 y $33 \%$. Los datos obtenidos en estudios abiertos se han confirmado en el ensayo clínico GAIN (6), estudio doble ciego, multicéntrico, controlado con placebo con 325 pacientes con EC que han recibido previamente IFX y que presentaron pérdida de respuesta o intolerancia. El tratamiento de inducción se realizó con 160/80 mg y la respuesta se valoró a las 4 semanas: un $21 \%$ de los pacientes entró en remisión y un $38 \%$ presenta respuesta parcial. En los estudios comentados no se hallaron diferencias en la eficacia en relación al motivo que propició la suspensión del IFX. En nuestra serie las tasas de remisión y respuesta parcial obtenidas son del 20 y $66,7 \%$ respectivamente, datos con un valor relativo por las obvias limitaciones de nuestro estudio (tamaño muestral y ausencia de grupo control).

El adalimumab también ha demostrado su eficacia en mantener la respuesta clínica cuando se compara con placebo en estudios controlados como el CLASSIC II $(3,5)$ y en estudios abiertos (12). El estudio CHARM (3) es un ensayo clínico controlado con una fase abierta para pacientes naïve o no para antiTNF $\alpha$. Todos los pacientes $(\mathrm{n}=499)$ reciben tratamiento de inducción con $80 / 40 \mathrm{mg}$, y posteriormente se randomizan para recibir adalimumab $40 \mathrm{mg}$ cada dos semanas, $40 \mathrm{mg}$ semanal o placebo. El porcentaje de pacientes en remisión a las 56 semanas fue del $47 \%$ en el grupo de adalimumab semanal y del $41 \%$ en el grupo de adalimumab cada 2 semanas, sin objetivarse diferencias entre ambas pautas. El estudio CHARM no encontró diferencias en los porcentajes de remisión y respuesta en función de si los pacientes habían recibido previamente IFX o no. En nuestro estudio un $72 \%$ de los pacientes con enfermedad luminal mantienen la respuesta a las 48 semanas y el $54 \%$ a las 72 semanas lo que confirma la eficacia de adalimumab como tratamiento de mantenimiento en esta indicación. Un $27 \%$ de los pacientes requirieron acortar el intervalo entre dosis para mantener la respuesta.

Con respecto a los datos que disponemos en relación con la enfermedad perianal en el estudio CLASSIC I (4) (enfermedad fistulosa perianal y enterocutánea) no se objetivan diferencias en eficacia entre adalimumab y placebo. En el ya citado estudio CHARM (3) se realiza un sub-análisis en los 130 pacientes $(15,2 \%)$ que presentaron fístulas perianales o enterocutáneas. A las 56 semanas el cierre completo de las fístulas perianales se produce en el $30 \%$ de los pacientes del grupo tratado con adalimumab frente al $13 \%$ en el grupo con placebo $(p=0,043)$. En el estudio no controlado de Hinojosa y cols. (13) un $64 \%$ de los 22 pacientes con enfermedad fistulosa perianal presentan, a la semana, 4 remisión
$(23 \%)$ o respuesta parcial $(41 \%)$. A la semana 20 la mitad de los pacientes mantienen la remisión $(12,5 \%)$ o la respuesta $(37,5 \%)$. En nuestro estudio 5 de los 6 pacientes tratados por enfermedad fistulosa perianal desarrollan remisión o respuesta con el tratamiento de inducción y todos ellos mantienen la respuesta con una mediana de tiempo de seguimiento de 15 meses.

Un estudio reciente, unicéntrico y con similar número de pacientes que el nuestro, corrobora la eficacia y seguridad del adalimumab en el contexto de la EC refractaria al tratamiento convencional y con administración previa de IFX (17).

La seguridad del adalimumab en la EC (3-6) es similar a la observada con otros antiTNF $\alpha(7,8)$ y en los estudios realizados en artritis reumatoide (18). En nuestra serie los efectos secundarios fueron leves y bien tolerados y los que presentaron una mayor gravedad con suspensión del tratamiento estaban relacionados con un mal control de la enfermedad (una obstrucción intestinal). Ningún paciente de nuestro grupo presentó infecciones graves ni reacciones de hipersensibilidad al fármaco.

Con las limitaciones evidentes del estudio (ausencia de grupo control, limitado número de pacientes) podemos decir que los resultados obtenidos en nuestra serie confirman en un contexto de práctica clínica habitual, que el adalimumab es eficaz y seguro en el tratamiento de la EC.

\section{BIBLIOGRAFÍA}

1. Papadakis KA. Adalimumab for the treatment of Crohn's disease. Expert Rev Clin Immunol 2006; 2(1): 11-5.

2. Martinez-Montiel MP, MT Munoz-Yague. Biologic therapies for chronic inflammatory bowel disease. Rev Esp Enferm Dig 2006; 98(4): 265-91.

3. Colombel JF, Sandborn WJ, Rutgeerts P, et al. Adalimumab for maintenance of clinical response and remission in patients with Crohn's disease: the CHARM trial. Gastroenterology 2007; 132(1): 52-65.

4. Hanauer SB, Sandborn WJ, Rutgeerts P, et al. Human anti-tumor necrosis factor monoclonal antibody (adalimumab) in Crohn's disease: the CLASSIC-I trial. Gastroenterology 2006; 130(2): 323-33.

5. Sandborn WJ, Hanauer SB, Rutgeerts $\mathrm{P}$, et al. Adalimumab for maintenance treatment of Crohn's disease: results of the CLASSIC II Trial. Gut 2007; 56: 1232-9.

6. Sandborn WJ, Rutgeerts P, Enns R, et al. Adalimumab Induction Therapy for Crohn's disease previously treated with infliximab. A randomized trial. Ann Intern Med 2007; 146(12): 829-38.

7. Hanauer SB, Feagan BG, Lichtenstein GR, et al. Maintenance infliximab for Crohn's disease: the ACCENT I trial. Lancet 2002; 359(9317): 1541-9.

8. Sands BE, Anderson FH, Bernstein CN, et al. Infliximab maintenance therapy for fistulizing Crohn's disease: the ACCENT II trial. N Engl J Med 2004; 350(9): 876-85.

9. Baert $\mathrm{F}$, Noman $\mathrm{M}$, Vermeire $\mathrm{S}$, et al. Influence of immunogenicity on the long-term efficacy of infliximab in Crohn's disease. N Engl J Med 2003; 348(7): 601-8.

10. Vermeire S, Noman M, van Assche G, et al. The effectiviness of concomitant immunosuppressive therapy to suppress formation of antibodies to infliximab in crohn's disease. Gut 2007; 56(9): 1226-31.

11. Best WR. Predictig the Crohn's disease activity index from the Harvey-Bradshaw Index. Inflamm Bowel Dis 2006; 12(4): 304-10. 
12. Peyrin-Biroulet L, Laclotte C, Bigard MA. Adalimumab maintenance therapy for Crohn's disease with intolerance or lost response to infliximab: an open-label study. Aliment Pharmacol Ther 2007; 25(6): 675-80.

13. Hinojosa J, Gomollon F, García S, et al. Efficacy and safety of shortterm adalimumab treatment in patients with active Crohn's disease who lost response or showed intolerance to infliximab: a prospective, open-label, multicentre trial. Aliment Pharmacol Ther 2007; 25(4): 409-18.

14. Papadakis KA, Shaye OA, Vasiliauskas EA, et al. Safety and efficacy of adalimumab (D2E7) in Crohn's disease patients with an attenuated response to infliximab. Am J Gastroenterol 2005; 100(1): 75-9.

15. Youdim A, Vasiliauskas EA, Targan SR, et al. A pilot study of adali- mumab in infliximab-allergic patients. Inflamm Bowel Dis 2004; 10(4): 333-8.

16. Sandborn WJ, Hanauer S, Loftus EV Jr, et al. An open-label study of the human anti-TNF monoclonal antibody adalimumab in subjects with prior loss of response or intolerance to infliximab for Crohn's disease. Am J Gastroenterol 2004; 99(10): 1984-9.

17. Ho GT, Smith L, Aitken S, Lee HM, Ting T, Fennell J. The use of adalimumab in the management of refractory Crohn's disease. Aliment Pharmacol Ther 2008; 27(4): 308-15.

18. Schiff MH, Burmester GR, Kent JD, et al. Safety analyses of adalimumab (HUMIRA) in global clinical trials and US postmarketing surveillance of patients with rheumatoid arthritis. Ann Rheum Dis 2006; 65(7): 889-94. 